\title{
Molecular epidemiology and clinical spectrum of hereditary spastic paraplegia in the Japanese population based on comprehensive mutational analyses
}

Hiroyuki Ishiura, Yuji Takahashi, Toshihiro Hayashi, Kayoko Saito, Hirokazu Furuya, Mitsunori Watanabe, Miho Murata, Mikiya Suzuki, Akira Sugiura, Setsu Sawai, Kazumoto Shibuya, Naohisa Ueda, Yaeko Ichikawa, Ichiro Kanazawa, Jun Goto and Shoji Tsuji

Journal of Human Genetics (2016) 61, 363-364; doi:10.1038/jhg.2015.159

Correction to: Journal of Human Genetics (2014) 59, 163-172; doi:10.1038/jhg.2013.139; published online 23 January 2014

Incorrect description in Table 2 in the above-mentioned article was noticed after its publication.

1. In the title for Table 2, 'Mutation and clinical summary of Japanese HSP patients' should be 'Mutations and clinical summaries of Japanese HSP patients'.

2. The nucleotide description for p.R562Q of SPAST should be c.1685G $>$ A instead of c.1688G $>$ A.

3. The position of deletion of SPAST (intron 16 3' region) should be Chr2: 32231942_32290423 which causes 58 482-bp deletion instead of Chr2: 32290425-32231940.
4. The position of the REEP1 deletion (intron $3 \sim$ intron 5) should be Chr2:86326359_86338423 which causes 12 065-bp deletion instead of Chr2: $86326358-86338428$.

5. The nucleotide description for p.K1614* of SPG11 should be c. $4840 \mathrm{~A}>\mathrm{T}$ instead of c. $4840 \mathrm{~T}>\mathrm{A}$.

6. In the description of SPAST deletions involving intron16 to 3'-UTR and exon 4 to intron 7, 'Chr2' should be added to clarify the position of the deletions.

7. The incorrect description of the foot note 'All the patients presented the pure form.' should be deleted.

These correction does not alter the results and their interpretation as discussed in the paper.

We apologize for this mistake. 
Table 2 Mutations and clinical summaries of Japanese HSP patients

\begin{tabular}{|c|c|c|c|c|c|c|c|c|}
\hline & Mutation & Amino-acid change & $\begin{array}{l}\text { Detecting } \\
\text { method }\end{array}$ & Family history & $\begin{array}{l}\text { Age at onset of } \\
\text { index patient }\end{array}$ & $\begin{array}{l}\text { Clinical } \\
\text { phenotype }\end{array}$ & & References \\
\hline \multicolumn{9}{|l|}{ SPG4 } \\
\hline Exon 1 & c. $139 \mathrm{~A}>\mathrm{T}$ & p.K47* & $\mathrm{R}$ & $A D$ & $40 s$ & Pure & Novel & \\
\hline Exon 1 & c. $155 \mathrm{~A}>\mathrm{G}$ & p.Y52C & $\mathrm{R}$ & $\begin{array}{l}\text { Sporadic with } \\
\text { consanguineous } \\
\text { parents }\end{array}$ & 49 у.о. & Pure & Novel & \\
\hline Exon 1 & c. $283 \_323 \mathrm{del}$ & p.A95Afs & D & $A D$ & 40 у.о. & Pure & Novel & \\
\hline Exon 1 & c.343_352 dup & p.V118Afs & $\mathrm{D}$ & $A D$ & 35 у.о. & Pure & Novel & \\
\hline Exon 2 & c. $422-425$ delAGAA & p.Q141fs & $\mathrm{D}$ & $A D$ & 36 у.о. & Pure & Novel & \\
\hline Exon 2 & c. $422-425$ delAGAA & p.Q141fs & $\mathrm{D}$ & $A D$ & 51 у.о. & Pure & Novel & \\
\hline Exon 2 & c. $422-425$ delAGAA & p.Q141fs & D & Sporadic & 35 у.о. & Pure & Novel & \\
\hline Exon 3 & c. $532 \mathrm{C}>\mathrm{T}$ & p.Q178* & $\mathrm{R}$ & $A D$ & 33 у.о. & Pure & Novel & \\
\hline Exon 5 & c. $734 \mathrm{C}>\mathrm{G}$ & p.S245* & $\mathrm{R}$ & $A D$ & Teens & Pure & Known & 35 \\
\hline Exon 5 & c. $838 \mathrm{C}>\mathrm{T}$ & p.Q280* & $\mathrm{R}$ & $A D$ & $\sim 6$ у.о. & Pure & Novel & \\
\hline Exon 6 & c.871 delG & p.G291Vfs & D & $A D$ & 20 y.o. & Pure & Novel & \\
\hline Intron 6 & c. $1005-2 A>G$ & IVS6-2A > G & $\mathrm{R}$ & $A D$ & 2 у.о. & Pure & Known & 4 \\
\hline Exon 7 & c. 1014 delT & p.A338Afs & D & $A D$ & $40 \mathrm{~s}$ & Pure & Novel & \\
\hline Exon 8 & c. $1105 \mathrm{~A}>\mathrm{C}$ & р.T369P & $\mathrm{R}$ & $A D$ & 38 у.о. & Pure & Novel & \\
\hline Exon 8 & c. $1141 \mathrm{C}>\mathrm{T}$ & p.F381L & $\mathrm{R}$ & Sporadic & $<6$ у.о. & Pure & Known & 4 \\
\hline Exon 8 & c. $1141 \mathrm{C}>\mathrm{T}$ & p.F381L & $\mathrm{R}$ & $A D$ & Late 50 s & Pure & Known & 4 \\
\hline Intron 8 & c. $1173+1 \mathrm{G}>\mathrm{A}$ & IVS $8+1 \mathrm{G}>\mathrm{A}$ & $\mathrm{R}$ & $A D$ & 46 у.о. & Pure & Known & 3 \\
\hline Exon 11 & c. $1378 \mathrm{C}>\mathrm{T}$ & p.R460C & $\mathrm{R}$ & $A D$ & 27 y.o. & Pure & Known & 36 \\
\hline Exon 12 & c. $1426 \_1427$ delGG & p.G476Rfs & D & $A D$ & 39 у.о. & Pure & Novel & \\
\hline Intron 12 & c. $1493+2 \mathrm{~T}>\mathrm{C}$ & IVS12+2T >C & $\mathrm{R}$ & Sporadic & 40 у.о. & Pure & Known & 35 \\
\hline Exon 13 & c. $1504 \mathrm{~A}>\mathrm{T}$ & p.K502* & $\mathrm{R}$ & $A D$ & 30 у.о. & Pure & Novel & \\
\hline Exon 13 & c. $1507 \mathrm{C}>\mathrm{T}$ & p.R503W & $\mathrm{R}$ & $A D$ & $\sim 10$ y.o. & Pure & Known & 37 \\
\hline Exon 15 & c. 1646 insT & p.L549Lfs & D & $A D$ & 34 у.о. & Pure & Novel & \\
\hline Exon 15 & c. 1646 insT & p.L549Lfs & $\mathrm{D}$ & $A D$ & 47 y.o. & Pure & Novel & \\
\hline Exon 15 & c. $1646 \mathrm{~T}>\mathrm{C}$ & p.L549P & $\mathrm{R}$ & $A D$ & $<15$ y.o. & Pure & Novel & \\
\hline Exon 15 & c. $1685 \mathrm{G}>\mathrm{A}$ & p.R562Q & $\mathrm{R}$ & $A D$ & $\sim 10$ у.о. & Pure & Known & 38 \\
\hline Exon 17 & c. $1741 \mathrm{C}>\mathrm{T}$ & p.R581* & $\mathrm{R}$ & $A D$ & 14 у.о. & Pure & Known & 39 \\
\hline Promoter intron 1 & $\begin{array}{l}\text { del Chr2: } 32136286-32145830 \\
\text { (9545 bp) }\end{array}$ & Del ex1 & $\mathrm{aCGH}$ & $A D$ & 40 y.o. & Pure & Novel & \\
\hline Intron $1 \sim 3^{\prime}$ region & $(>170 \mathrm{~kb})$ & Del ex2-17 & aCGH & Affected sibling & 24 у.о. & Pure & Novel & \\
\hline $\begin{array}{l}\text { Intron } 16 \sim 3^{\prime} \\
\text { region }\end{array}$ & $\begin{array}{l}\text { del Chr2: } 32231942-32290423 \\
\text { (58 } 482 \text { bp) }\end{array}$ & Del ex17 & aCGH & $A D$ & 58 у.о. & Pure & Novel & \\
\hline Intron $16 \sim 3^{\prime}$-UTR & $\begin{array}{l}\text { del Chr2: } 32229622-32234715 \\
\text { (5094 bp) }\end{array}$ & Del ex17 & aCGH & $A D$ & 52 y.o. & Pure & Novel & \\
\hline Exon 4 intron 7 & $\begin{array}{l}\text { dup Chr2: } 32177411-32199467 \\
\text { (22 } 057 \text { bp)+insAGT }\end{array}$ & $\begin{array}{l}\text { Tandem duplication } \\
\text { (part of ex4-ex7) }\end{array}$ & aCGH & $A D$ & $<6$ у.о. & Pure & Novel & \\
\hline \multicolumn{9}{|l|}{ SPG3A } \\
\hline Exon 12 & c. $1243 \mathrm{C}>\mathrm{T}$ & p.R415W & $R$ & $A D$ & 12 у.о. & Pure & Known & 40 \\
\hline Exon 12 & c. $1483 \mathrm{C}>\mathrm{T}$ & p.R495W & $\mathrm{R}$ & Sporadic & $\sim 12$ у.о. & Pure & Known & 41 \\
\hline \multicolumn{9}{|l|}{ SPG8 } \\
\hline Exon 13 & c. $1749 \mathrm{~A}>\mathrm{C}$ & p.R583S & $\mathrm{R}$ & $A D$ & 50 у.о. & Pure & Novel & \\
\hline $\begin{array}{l}\text { Intron } \\
10 \sim \text { exon } 15\end{array}$ & $\begin{array}{l}\text { del Ch8: } 126138189-126142822 \\
\text { (4634 bp) }\end{array}$ & Del exon 11-15 & $\mathrm{aCGH}$ & $A D$ & 64 y.o. & Pure & Novel & \\
\hline \multicolumn{9}{|l|}{ SPG17 } \\
\hline Exon 2 & c. $107 \mathrm{G}>\mathrm{A}$ (c. $299 \mathrm{G}>\mathrm{A}$ ) & p.C36Y (p.C100Y) & $\mathrm{R}$ & $A D$ & $\sim 10$ у.о. & Complicated & Novel & \\
\hline \multicolumn{9}{|l|}{ SPG31 } \\
\hline Exon 2 & c.87 insA & p.K30Kfs & D & $A D$ & 8 у.о. & Pure & Novel & \\
\hline Intron 3 intron 5 & $\begin{array}{l}\text { del Ch2: } 86326359-86338423 \\
\text { (12 } 065 \text { bp) }\end{array}$ & Del exon 4-5 & aCGH & $A D$ & 12 у.о. & Pure & Novel & \\
\hline \multicolumn{9}{|l|}{ SPG11 } \\
\hline Intron 18 & c. $3291+1 G>T$ & $\begin{array}{l}\text { IVS18+1G- }>\text { T } \\
\text { (homozygous) }\end{array}$ & $\mathrm{D}$ & $\begin{array}{l}\text { Consanguinity } \\
\text { affected siblings }\end{array}$ & 20 у.о. & Complicated & Known & 42 \\
\hline Intron 18 & c. $3291+1 G>T$ & $\begin{array}{l}\text { IVS18+1G- }>\text { T } \\
\text { (homozygous) }\end{array}$ & D & $\begin{array}{l}\text { Consanguinity } \\
\text { affected sibling }\end{array}$ & 25 у.о. & Complicated & Known & 42 \\
\hline $\begin{array}{l}\text { Intron } 8 \text { and } \\
\text { intron } 38\end{array}$ & $\begin{array}{l}\text { c. } 1735+2 \text { delT, } \\
\text { c. } 6999+5 \text { delG }\end{array}$ & $\begin{array}{l}\text { IVS8+2 delT, } \\
\text { IVS38+5 delG }\end{array}$ & $\begin{array}{l}D \\
D\end{array}$ & Sporadic & 22 у.о. & Complicated & Novel & \\
\hline Exon 20 and & c. $3491 \mathrm{G}>\mathrm{A}$ & p.W1164*, & $D, D$ & Sporadic & 18 у.о. & Complicated & Novel & \\
\hline $\begin{array}{l}\text { exon } 28 \\
\text { Intron } 7 \text { intron } 8\end{array}$ & c.4840 A>T & p.K1614* & & & & & & \\
\hline $\begin{array}{l}\text { Intron } 7 \sim \text { intron } 8 \\
\text { and exon25 }\end{array}$ & $\begin{array}{l}\text { del Ch15: } 42709367-42715955 \\
\text { (6589 bp) c.4426 insAT }\end{array}$ & Del exon 8, p.C1476Yfs & $\begin{array}{l}\text { aCGH } \\
\text { D }\end{array}$ & Affected sibling & 2 у.о. & Complicated & Novel & \\
\hline \multicolumn{9}{|l|}{ SPG21 } \\
\hline Exon 4 & c. $322 \mathrm{G}>\mathrm{C}$ & p.A108P (homozygous) & $\mathrm{R}$ & Familial & 60 у.о. & Complicated & Novel & \\
\hline
\end{tabular}

Abbreviations: aCGH, array-based comparative genomic hybridization analysis; AD, autosomal dominant; D, direct nucleotide sequence analysis; Del, deletion; dup, duplication; R, resequencing microarray analysis; UTR, untranslated region; y.o., years old.

+1 of nucleotides is the first A of the start codon (ATG). The NCBI36/hg18 assembly is used as the reference genome. 